\title{
Efficacy of temozolomide or dacarbazine in combination with an anthracycline for rescue chemotherapy in dogs with Iymphoma
}

\author{
Nikolaos G. Dervisis, Dvm; Pedro A. Dominguez, DVM; Luminita Sarbu, DVM, PhD; \\ Rebecca G. Newman, DVM, MS; Casey D. Cadile, DvM; Christine N. Swanson, DVM; \\ Barbara E. Kitchell, DVM, PhD, DACVIM
}

\begin{abstract}
Objective - To compare results of treatment with temozolomide or dacarbazine, in combination with an anthracycline, in dogs with relapsed or refractory lymphoma.

Design—Nonrandomized, controlled clinical trial.

Animals — 63 dogs with relapsed or refractory lymphoma.

Procedures - Chemotherapy was administered in 21-day cycles. A combination of temozolomide and an anthracycline (doxorubicin or dactinomycin) was administered to 21 dogs and a combination of dacarbazine and an anthracycline was administered to 42 dogs. Efficacy and toxicoses were assessed.

Results - Thirteen of the $18(72 \%)$ dogs treated with the temozolomide-anthracycline combination and 25 of the $35(71 \%)$ dogs treated with the dacarbazine-anthracycline combination had a complete or partial response. Median duration of response to rescue chemotherapy was 40 days (range, 0 to 217 days) for dogs in the temozolomide group and 50 days (range, 0 to 587 days) for dogs in the dacarbazine group. The incidence of high-grade hematologic toxicoses was significantly higher among dogs in the dacarbazine group than among dogs in the temozolomide group, but the incidence of gastrointestinal tract toxicoses was not significantly different between groups. There were no significant differences between groups in regard to proportion of dogs with a complete or partial response, duration of response to rescue chemotherapy, survival time following rescue chemotherapy, or overall survival time.

Conclusions and Clinical Relevance-Both combinations had promise in the treatment of dogs with relapsed or refractory lymphoma, although administration of temozolomide was more convenient than administration of dacarbazine and caused fewer hematologic toxicoses. (J Am Vet Med Assoc 2007;231:563-569)
\end{abstract}

\begin{abstract}
$\mathrm{T}$ reatment of relapsed or refractory lymphoma in dogs often involves administration of an anthracycline, such as doxorubicin or dactinomycin, in combination with an alkylating agent. Dacarbazine is an alkylating agent that has been used in the treatment of relapsed round cell tumors, high-grade sarcomas, and malignant melanomas, ${ }^{1}$ and results of in vitro and in vivo studies ${ }^{2,3}$ suggest that dacarbazine acts synergistically with anthracyclines in humans. Dacarbazine has been administered in combination with doxorubicin or dactinomycin for treatment of relapsed lymphoma in $\operatorname{dogs},{ }^{1,4}$ and this combination appears to have moderate antineoplastic activity. However, its use has been limited by the requirement that dacarbazine be administered as a slow IV infusion over 8 hours or as a bolus by slow IV injection once daily for 5 consecutive days every 3 weeks. ${ }^{5,6}$ In addition, dacarbazine has been associated with clinically important gastrointestinal tract toxicoses, both acute and delayed, and hematologic toxicoses, including neutrope-
\end{abstract}

From the Center for Comparative Oncology, College of Veterinary Medicine, Michigan State University, East Lansing, MI 48824.

Presented in part at the 26th Annual Veterinary Cancer Society

Meeting, Callaway Gardens, Ga, October 2006.

Address correspondence to Dr. Dervisis. nia and thrombocytopenia, and accidental extravasation may cause local irritation and pain..$^{1,4}$

Temozolomide is a recently developed imidazotetrazine derivative that is essentially an oral formulation of dacarbazine. ${ }^{7}$ Whereas dacarbazine must be activated by the cytochrome P-450 system in the liver, temozolomide is metabolized to its active form by a nonenzymatic pathway in the blood. ${ }^{8,9}$ Thus, we speculate that the response to treatment would be better with temozolomide, compared with dacarbazine, because of elimination of the variable introduced by cytochrome P-450 activation necessary for dacarbazine activity. Further, we hypothesize that the toxicity of dacarbazine is influenced by pharmacogenomic factors introduced by variations in cytochrome P-450 activity among individuals and that toxicity would thus be minimized by use of temozolomide. ${ }^{8,10}$ Finally, because temozolomide is hydrolyzed to a common intermediate of the dacarbazine activation pathway, we suspect that temozolomide might have pharmacokinetic and clinical benefits in relation to dacarbazine.

The purpose of the study reported here, therefore, was to compare results of treatment with temozolomide or dacarbazine, in combination with an anthracycline, in dogs with relapsed or refractory lymphoma. 


\section{Materials and Methods}

Dogs-Dogs examined at the Michigan State University Animal Cancer Care Clinic between January 2004 and December 2006 because of relapsed or refractory lymphoma were eligible for inclusion in the study. Dogs with relapsed lymphoma were included only if they had received induction treatment with a multidrug chemotherapy protocol that included doxorubicin; dogs with refractory lymphoma were included only if they had not responded to a standard induction chemotherapy protocol. In addition, dogs were included only if they had measurable disease and the diagnosis had been confirmed on the basis of results of histologic or cytologic examination. Dogs with nonneoplastic comorbid conditions were excluded from the study.

Group assignment-Two treatment groups (temozolomide and an anthracycline vs dacarbazine and an anthracycline) were included in the study. At the time of the study, the cost of treating a dog with a combination of temozolomide and an anthracycline was approximately twice the cost of treating a dog with a combination of dacarbazine and an anthracycline. Therefore, dogs were not randomly assigned to treatment groups. Rather, treatment group assignments were made on the basis of owner cost concerns. Owners of all dogs included in the study provided informed consent, and the study was performed in compliance with guidelines for research in animals established by the Michigan State University All-University Committee on Animal Use and Care.

Within each treatment group, dogs were treated with doxorubicin or dactinomycin on the basis of perceived risk of developing doxorubicin-related cardiotoxicosis. Patients evaluated as being at high risk for cardiotoxicosis were switched to dactinomycin instead of doxorubicin. Dogs were considered to have a high risk of developing doxorubicin-related cardiotoxicosis if systolic fractional shortening determined echocardiographically was $<25 \%$ or if total prior cumulative doxorubicin dose was $\geq 180 \mathrm{mg} / \mathrm{m}^{2}$.

Treatment protocol-Doxorubicin ${ }^{\mathrm{a}}$ was diluted in 50 to $100 \mathrm{~mL}$ of $0.9 \% \mathrm{NaCl}$ and administered through an indwelling IV catheter over 20 minutes at a dose of 30 $\mathrm{mg} / \mathrm{m}^{2}$. Dactinomycin ${ }^{\mathrm{b}}$ was also diluted in 50 to $100 \mathrm{~mL}$ of $0.9 \% \mathrm{NaCl}$ and administered through an indwelling IV catheter over 20 minutes, but at a dose of $0.6 \mathrm{mg} / \mathrm{m}^{2}$. Dacarbazine ${ }^{\mathrm{c}}$ was administered immediately after anthracycline administration through the same catheter at a dose of $800 \mathrm{mg} / \mathrm{m}^{2}$; the calculated dose was diluted in $0.9 \% \mathrm{NaCl}(17.6 \mathrm{~mL} / \mathrm{kg}[8 \mathrm{~mL} / \mathrm{b}])$ and administered over 8 hours. Dogs were treated with dexamethasone sodium phosphate (4 mg, IV) and butorphanol $(0.4 \mathrm{mg} / \mathrm{kg}[0.18$ $\mathrm{mg} / \mathrm{lb}], \mathrm{IM})$ before each dacarbazine treatment, and metoclopramide $(0.5 \mathrm{mg} / \mathrm{kg}[0.23 \mathrm{mg} / \mathrm{lb}], \mathrm{PO})$ was dispensed to be administered as needed at home. Temozolomided was dispensed to participating owners to be administered at home. Owners were instructed to wear latex gloves when handling the drug, to not open the capsules, and to not allow their animal to chew the capsules. The initial intended dosage of temozolomide was $60 \mathrm{mg} / \mathrm{m}^{2}, \mathrm{PO}$, every 24 hours for 5 consecutive days, starting on the same day as anthracycline administration. For each successive group of 3 dogs, the dose was increased by a factor of $10 \mathrm{mg} / \mathrm{m}^{2}$, up to an intended target dose of $100 \mathrm{mg} / \mathrm{m}^{2}$, as long as none of the 3 dogs in the group developed toxicoses that required hospitalization. Because temozolomide was supplied as 5-, 20-, 100-, and 250-mg capsules, the calculated dose of temozolomide was rounded to the nearest $5 \mathrm{mg}$; reformulation was not done. Owners were instructed to administer temozolomide on an empty stomach and to feed their dog 30 minutes later. Metoclopramide $(0.5 \mathrm{mg} /$ $\mathrm{kg}, \mathrm{PO}$ ) was administered 1 hour before temozolomide to prevent vomiting and as needed throughout the treatment period. The chemotherapy treatment was repeated every 21 days in dogs that had a clinical response of at least 21 days' duration.

Evaluation of response and toxicoses-In all dogs, a CBC and complete physical examination were performed on the day of anthracycline administration and 7 and 21 days later at the Animal Cancer Care Clinic or by the referring veterinarian. In addition, thoracic radiography and abdominal ultrasonography were performed after every 2 to 3 treatment cycles in dogs that continued to respond. Any evidence of lymphadenopathy or ultrasonographic lesions was investigated by means of cytologic examination of fine-needle aspirates. Dogs were considered lost to follow-up when not returned for scheduled recheck examinations and the veterinarian or owner could not be contacted despite repeated attempts; dogs lost to follow-up were censored.

Tumor response was determined each time dogs were examined. A complete response was defined as disappearance of all measurable disease for 21 days. A partial response was defined as $>50 \%$ but $<100 \%$ reduction in measurable disease for 21 days. Stable disease was defined as $<50 \%$ reduction in measurable disease for 21 days with no appearance of new lesions during that period. Progressive disease was defined as $>25 \%$ increase in measurable disease or appearance of new lesions. Transient decreases in measurable disease that persisted for $<21$ days were defined as progressive disease.

Toxicoses were identified on the basis of history and results of physical examinations and $\mathrm{CBCs}$. Criteria established by the Veterinary Cooperative Oncology Group $^{11}$ were used to grade toxicoses.

Statistical analysis-Duration of first remission was defined as the time from initial administration of the original induction chemotherapy protocol until relapse. Duration of response to rescue chemotherapy was defined as the time from completion of the initial cycle of rescue chemotherapy until progression of disease. Survival time following rescue chemotherapy was defined as the time from completion of the initial cycle of rescue chemotherapy until death. Overall survival time was defined as the time from initial administration of the original induction chemotherapy protocol until death. The Kaplan-Meier survival analysis method was used to estimate response and survival time curves following treatment. The log-rank test was used to compare Kaplan-Meier curves between treatment groups (temozolomide treatment group vs dacarbazine 
treatment group), between subgroups (doxorubicin vs dactinomycin vs doxorubicin followed by dactinomycin subgroup), and between responding and nonresponding dogs. Continuous variables (age and body weight) were tested by use of the D'Agostino-Pearson test to determine whether they were normally distributed. Continuous variables that were found to be normally distributed were compared between treatment groups by means of a 2 -sample $t$ test. The Pearson $\chi^{2}$ test was used to determine whether the distribution of categoric variables (sex, stage, substage, immunophenotype, and presence of hypercalcemia) differed between treatment groups. The Cox proportional-hazards regression method was used to determine whether potential risk factors (ie, sex, age, body weight, stage, substage, immunophenotype, and presence of hypercalcemia) were associated with duration of response to rescue chemotherapy or survival time following rescue chemotherapy. The potential association between dosage of temozolomide and duration of response to rescue chemotherapy was examined by use of the log-rank test for trends. The $\chi^{2}$ test with Yates' correction for continuity was used to determine whether treatment-related toxicoses were associated with treatment group.

All reported $P$ values are 2 -sided. Values of $P<0.05$ were considered significant. Statistical analyses were performed with standard software. ${ }^{\mathrm{e}}$

\section{Results}

Patients-Sixty-three dogs were enrolled in the study. Twenty-one dogs were treated with a combination of temozolomide and an anthracycline, and 42 were treated with a combination of dacarbazine and an anthracycline. A total of 55 cycles of the temozolomideanthracycline protocol were administered, of which 41 included doxorubicin and 14 included dactinomycin. A total of 119 cycles of the dacarbazine-anthracycline protocol were administered, of which 48 included doxorubicin and 71 included dactinomycin.

Mean age of dogs in the temozolomide treatment group (mean $\pm \mathrm{SD}, 10.1 \pm 3.2$ years) was not significantly $(P=0.47)$ different from mean age of dogs in the dacarbazine treatment group ( $8.5 \pm 2.8$ years). Similarly, mean body weight of dogs in the temozolomide treatment group $(22.4 \pm 12.3 \mathrm{~kg}[49.3 \pm 27.1 \mathrm{lb}])$ was not significantly $(P=0.51)$ different from mean body weight of dogs in the dacarbazine treatment group (34.2 \pm 10.9 $\mathrm{kg}[75.2 \pm 24 \mathrm{lb}]$ ). Treatment groups did not differ with regard to sex, breed, initial stage, initial substage, immunophenotype, or presence of hypercalcemia at the time of initial examination (Table 1).

Previous treatment-Prior to enrollment in the present study, all dogs had been treated with a multidrug chemotherapy protocol (cyclophosphamide, doxorubicin, vincristine, prednisone, and L-asparaginase). Dogs in the dacarbazine treatment group had received a median of 5 chemotherapy drugs (range, 3 to 8 ). Dogs in the temozolomide treatment group had also received a median of 5 chemotherapy drugs (range, 3 to 10). All but 1 dog had received a combination of L-asparaginase, vincristine, cyclophosphamide, doxorubicin, and prednisone for induction chemotherapy. The remaining dog did not receive vincristine because of severe cholestasis and hypoalbuminemia. Median duration of first remission prior to enrollment in the present study was 151 days (range, 26 to 320 days) for dogs in the temozolomide treatment group, with 14 of the 21 (67\%) dogs relapsing during the initial induction protocol. Median duration of first remission prior to enrollment in the present study was 145 days (range, 16 to 321 days) for dogs in the dacarbazine treatment group, with 29 of the 42 (69\%) dogs relapsing during the initial induction protocol. The temozolomide-anthracycline combination was the first rescue chemotherapy protocol for 12 of the 21 dogs in the temozolomide treatment group, the second rescue chemotherapy protocol for 3 dogs, the third rescue chemotherapy protocol 5 dogs, and the fifth rescue chemotherapy protocol for $1 \mathrm{dog}$. The dacarbazine-anthracycline combination was the first rescue chemotherapy protocol for 34 of the 42 dogs in the dacarbazine treatment group, the second rescue chemotherapy protocol for 5 dogs, and the third rescue chemotherapy protocol for 3 dogs.

Temozolomide treatment group-Median number of treatment cycles for dogs in the temozolomide treatment group was 2 (range, 1 to 8). Median dosage of temozolomide was $92.6 \mathrm{mg} / \mathrm{m}^{2}$ (range, 60.6 to 103.9 $\mathrm{mg} / \mathrm{m}^{2}$ ), and median cumulative dose was $953 \mathrm{mg} / \mathrm{m}^{2}$ (range, 305.1 to $3,692 \mathrm{mg} / \mathrm{m}^{2}$ ).

Eighteen dogs were evaluated for response, while 3 dogs were lost to follow-up. Median duration of response to rescue chemotherapy for the 18 dogs in the temozolomide treatment group was 40 days (range, 0 to 217 days). Thirteen dogs ( $72 \%$ ) had a complete (n =9) or partial (4) response, and median duration of response for these dogs was 65 days (range, 23 to 217 days). The remaining 5 dogs (28\%) had progressive dis-

Table 1-Characteristics of 63 dogs with relapsed or refractory lymphoma enrolled in a study comparing results of rescue chemotherapy with temozolomide and an anthracycline (ie, doxorubicin or dactinomycin; $\mathrm{n}=21$ ) or dacarbazine and an anthracycline (42).

\begin{tabular}{|c|c|c|}
\hline \multirow[b]{2}{*}{ Variable } & \multicolumn{2}{|c|}{ No. of dogs } \\
\hline & Temozolomide group & Dacarbazine group \\
\hline \multicolumn{3}{|l|}{ Sex } \\
\hline Male & 9 & 21 \\
\hline Female & 12 & 21 \\
\hline \multicolumn{3}{|l|}{ Breed } \\
\hline Mixed & 6 & 11 \\
\hline Golden Retriever & 3 & 9 \\
\hline Labrador Retriever & 0 & 6 \\
\hline $\begin{array}{l}\text { Boxer } \\
\text { lat }\end{array}$ & 1 & 5 \\
\hline Other purebred & 11 & 11 \\
\hline \multicolumn{3}{|l|}{ Initial stage } \\
\hline III & 5 & 14 \\
\hline IV & 9 & 18 \\
\hline V & 7 & 10 \\
\hline \multicolumn{3}{|l|}{ Initial substage } \\
\hline a & 11 & 27 \\
\hline $\mathrm{b}$ & 10 & 15 \\
\hline \multicolumn{3}{|l|}{ Hypercalcemia at initial } \\
\hline Yes & 4 & 5 \\
\hline No & 17 & 37 \\
\hline \multicolumn{3}{|l|}{ Immunophenotype } \\
\hline B cell & 8 & 23 \\
\hline T cell & 7 & 7 \\
\hline Non-B-non-T cell & 0 & 2 \\
\hline Unknown & 6 & 10 \\
\hline
\end{tabular}


ease, with duration of response recorded as 0 days for all 5 dogs. Sixteen dogs received temozolomide in combination with doxorubicin, 3 dogs received temozolomide in combination with dactinomycin, and 2 dogs initially received temozolomide in combination with doxorubicin but were switched to dactinomycin after they reached a cumulative doxorubicin dose of $180 \mathrm{mg} / \mathrm{m}^{2}$. No significant $(P=0.76)$ difference was found between these 3 subgroups (doxorubicin vs dactinomycin vs doxorubicin followed by dactinomycin) with regard to duration of response.

Median survival time following rescue chemotherapy for the 18 dogs in the temozolomide treatment group was 72 days (range, 30 to 352 days), with 3 dogs still alive at the time of the study. Median overall survival time (ie, time from initial administration of the original induction chemotherapy protocol until death) was 230 days (range, 87 to 646 days). Median overall survival time for the 13 dogs that had a complete or partial response to rescue chemotherapy was 251 days (range, 89 to 646 days), whereas median survival time for the 5 dogs that did not have a complete or partial response to rescue chemotherapy was 166 days (range, 87 to 464 days). Median overall survival time was not significantly $(P=0.27)$ different between these 2 subgroups.

Dacarbazine treatment group-Median number of treatment cycles for dogs in the dacarbazine treatment group was 2 (range, 1 to 9). Median dose of dacarbazine was 800 $\mathrm{mg} / \mathrm{m}^{2}$ (range, 450 to $800 \mathrm{mg} / \mathrm{m}^{2}$ ), and median cumulative dose was $1,400 \mathrm{mg} / \mathrm{m}^{2}$ (range, 584 to $6,300 \mathrm{mg} / \mathrm{m}^{2}$ ).

Thirty-five dogs were evaluated for response, while 7 dogs were lost to follow-up. Median duration of response to rescue chemotherapy for the 35 dogs in the dacarbazine treatment group was 50 days (range, 0 to 587 days). Twenty-five dogs $(71 \%)$ had a complete $(n=22)$ or partial (3) response, and median duration of response for these dogs was 85 days (range, 34 to 587 days). Seventeen dogs received dacarbazine in combination with doxorubicin, 22 dogs received dacarbazine in combination with dactinomycin, and 3 dogs initially received dacarbazine in combination with doxorubicin but were switched to dactinomycin after they reached a cumulative doxorubicin dose of $180 \mathrm{mg} / \mathrm{m}^{2}$. No significant $(P=0.27)$ difference was found between these 3 subgroups (doxorubicin vs dactinomycin vs doxorubicin followed by dactinomycin) with regard to duration of the response.

Median survival time following rescue chemotherapy for the 35 dogs in the dacarbazine treatment group was 104 days (range, 10 to 587 days), with 4 dogs still alive at the time of the study. Median overall survival time was 238 days (range, 57 to 688 days). Median overall survival time for the 25 dogs that had a complete or partial response to rescue chemotherapy was 337 days (range, 127 to 688 days), whereas median survival time for the 10 dogs that did not have a complete or partial response to rescue chemotherapy was 105 days (range, 57 to 188 days). Median overall survival time was significantly $(P<0.001)$ different between these 2 subgroups.

Toxicoses-For dogs in both treatment groups, the most common toxicoses were hematologic and gastrointestinal. Overall, for dogs in the temozolomide treatment group, there were 15 episodes of grade I hematologic toxicosis, 6 episodes of grade II hematologic toxicosis, 2 episodes of grade III hematologic toxicosis (thrombocytopenia), and 1 episode of grade IV hematologic toxicosis (neutropenia). Only 1 dog required hospitalization as a result of hematologic toxicosis (neutropenic sepsis and sinusitis), and in 3 instances, the dosage of temozolomide was reduced because of hematologic toxicoses. Gastrointestinal tract toxicoses (nausea, vomiting, and diarrhea) that occurred were self-limiting, and no dogs required hospitalization. There were 14 episodes of grade I, 7 episodes of grade II, and 1 episode of grade IV (nausea) gastrointestinal tract toxicosis. In 2 dogs, treatment with temozolomide was discontinued because of the high cost. Two dogs became azotemic, 1 after the second treatment, and the other after the fifth treatment. The former dog also had preexisting heart disease and was receiving mexiletene and enalapril; this dog was euthanized because of progressive renal failure while in remission. The other dog that developed azotemia received another 3 cycles of treatment, after which the lymphoma relapsed; the azotemia did not progress during this period. Another dog had episodes of urinary incontinence 2 to 3 days after the first day of treatment with temozolomide. Results of microbial (bacterial, mycoplasmal, and ureoplasmal) culture were negative, and the dog regained urinary continence after every treatment cycle.

Overall, for dogs in the dacarbazine treatment group, there were 79 episodes of grade I hematologic toxicosis, 28 episodes of grade II hematologic toxicosis, 18 episodes of grade III hematologic toxicosis, and 17 episodes of grade IV hematologic toxicosis (neutropenia and thrombocytopenia). Eight dogs had to be hospitalized because of neutropenic sepsis and dehydration, and 1 dog had to be hospitalized because of neutropenic sepsis and petechiation secondary to thrombocytopenia. The latter dog was euthanized after 2 days of hospitalization because of toxicoses. Five of the dogs hospitalized because of neutropenia had concurrent grade III $(n=4)$ or grade IV (1) gastrointestinal tract toxicoses (nausea, vomiting, and diarrhea). In 16 instances, the dosage of dacarbazine was reduced because of hematologic toxicoses. In 1 dog, treatment with dacarbazine was discontinued, and in 2 dogs, treatment was changed to temozolomide because of toxicoses. There were 20 episodes of grade I, 21 episodes of grade II, 7 episodes of grade III, and 2 episodes of grade IV gastrointestinal tract toxicosis.

Dogs in the temozolomide treatment group had a total of 24 episodes of hematologic toxicosis ( 0.44 episodes/treatment), whereas dogs in the dacarbazine treatment group had a total of 142 episodes of hematologic toxicosis (1.19 episodes/treatment). The incidence of high-grade (ie, grade III or IV) hematologic toxicoses was significantly $(P<0.001)$ higher among dogs in the dacarbazine treatment group than among dogs in the temozolomide treatment group. However, the incidence of gastrointestinal tract toxicoses did not differ significantly between groups.

Risk factor analysis-There were no significant differences between treatment groups in regard to proportion of dogs with a complete or partial response, du- 


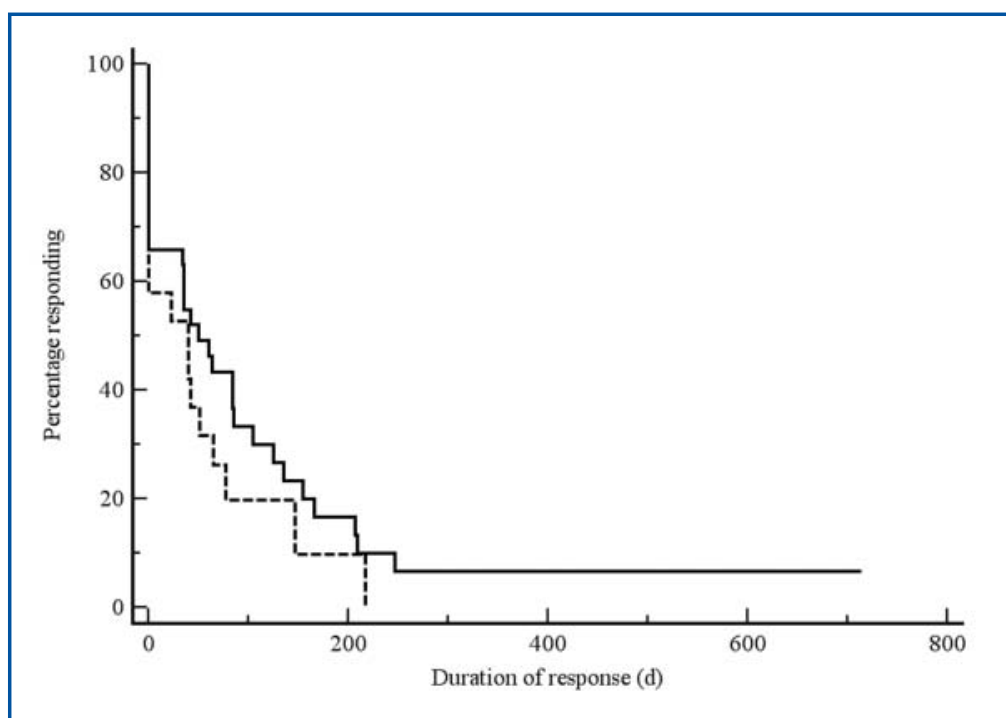

Figure 1-Kaplan-Meier curves of duration of response to rescue chemotherapy with temozolomide and an anthracycline (ie, doxorubicin or dactinomycin; $n=18$; dashed line) or dacarbazine and an anthracycline (35; solid line).

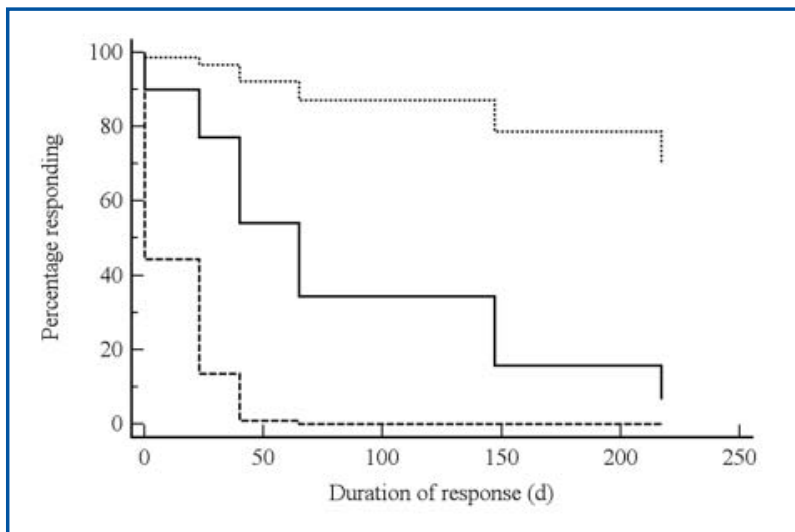

Figure 2-Kaplan-Meier curves of duration of response to rescue chemotherapy with temozolomide and an anthracycline in dogs with relapsed or refractory lymphoma grouped on the basis of initial stage (stage 3, dotted line; stage 4, solid line; stage 5, dashed line).

ration of response to rescue chemotherapy (Figure 1), survival time following rescue chemotherapy, or overall survival time. Sex, age, body weight, stage, substage, and hypercalcemia status were not found to be significantly associated with duration of response to rescue chemotherapy or overall survival time.

For dogs in the temozolomide treatment group, multivariate analysis indicated that stage was significantly $(P$ $=0.02)$ associated with duration of response to rescue chemotherapy (Figure 2$)$, that age $(P=0.02)$ and stage $(P=0.03)$ were significantly associated with survival time following rescue chemotherapy, and that immunophenotype was significantly $(P=0.02)$ associated with overall survival time. When dogs were grouped on the basis of anthracycline administration (doxorubicin vs dactinomycin vs doxorubicin followed by dactinomycin), there were no significant differences among groups with regard to proportion of dogs with a complete or partial response, duration of response to rescue chemotherapy, survival time foldacarbazine. lowing rescue chemotherapy, overall survival time, incidence of hematologic toxicoses, or incidence of gastrointestinal tract toxicoses.

For dogs in the dacarbazine treatment group, multivariate analysis indicated that body weight was significantly $(P=0.01)$ associated with survival time following rescue chemotherapy and that body weight $(P=$ $0.005)$, hypercalcemia status $(P=0.03)$, and immunophenotype $(P=0.04)$ were significantly associated with overall survival time. When dogs were grouped on the basis of anthracycline administration (doxorubicin vs dactinomycin vs doxorubicin followed by dactinomycin), there were no significant differences among groups with regard to proportion of dogs with a complete or partial response, duration of response to rescue chemotherapy, survival time following rescue chemotherapy, overall survival time, incidence of hematologic toxicoses, or incidence of gastrointestinal tract toxicoses.

\section{Discussion}

Results of the present study suggested that administration of temozolomide at a dose of up to $100 \mathrm{mg} / \mathrm{m}^{2}$ in combination with an anthracycline every 3 weeks appeared to be well tolerated in dogs with relapsed or refractory lymphoma. In particular, the incidence of hematologic toxicoses was significantly lower in dogs given the temozolomide-anthracycline combination, compared with dogs given a combination of dacarbazine and an anthracycline, although there was no difference in incidence of gastrointestinal tract toxicoses between treatment groups. Both combinations had promise in the treatment of dogs with relapsed or refractory lymphoma, although administration of temozolomide was more convenient and less toxic than administration of

The present study was primarily designed to evaluate the toxicity of temozolomide when administered in combination with an anthracycline, and tumor response was a secondary end point. Dacarbazine was used as the comparator because the active forms of temozolomide and dacarbazine are essentially identical with regard to mechanism of action. ${ }^{8}$ Bioavailability of temozolomide following oral administration in dogs is $100 \%$, and there is no clinically important metabolism or accumulation of the drug in the body. ${ }^{12}$ The main route of temozolomide excretion in dogs is through the kidneys, with minimal excretion taking place through the feces or respiratory tract. Dogs appear to be more sensitive to temozolomide than humans, in that the maximum nonlethal dose for dogs $\left(200 \mathrm{mg} / \mathrm{m}^{2}\right)$ is similar to the therapeutic dose for humans. ${ }^{13}$ In a chemoprotection study, ${ }^{14}$ dogs were given temozolomide at doses as high as $800 \mathrm{mg} / \mathrm{m}^{2}$, but the bone marrow was protected in these dogs by transplantation with stem cells transfected with specific drug resistance genes. In dogs given lethal doses, signs of potential CNS toxicosis were observed, including high body temperature, nausea, and vomiting. Toxic effects in dogs given nonlethal doses primarily involved the bone marrow, lymphoid tissues, 
testes, and gastrointestinal tract, as would be expected with an alkylating agent. No cardiovascular effects or renal abnormalities were detected in dogs treated with temozolomide in toxicology studies, and the carcinogenic potential of temozolomide appears to be similar to that of dacarbazine. ${ }^{13}$

In human studies, ${ }^{15,16}$ administration of temozolomide with food resulted in a $33 \%$ decrease in maximum serum concentration and a $9 \%$ decrease in the area under the concentration-versus-time curve. Although the clinical importance of these changes is unclear, it has been suggested that temozolomide be administered on an empty stomach. ${ }^{15,16}$ In the present study, therefore, we advised owners to give temozolomide on an empty stomach and to offer food 30 minutes later to allow for maximum absorption of the drug while limiting gastric irritation.

In the present study, dogs were assigned to treatment groups solely on the basis of the owner's ability to afford the more expensive treatment. We believe that this reduced the bias associated with allowing clinicians to assign cases to treatment groups. However, owners willing to invest more financially might have been more inclined to delay euthanasia in the event of treatment failure, adding some degree of bias to survival outcomes in our study. In contrast, dogs were assigned to receive doxorubicin or dactinomycin on the basis of objective criteria (ie, total cumulative dose of doxorubicin received and echocardiographic evidence of impaired systolic function). Dogs that reached a cumulative dose of doxorubicin of 180 $\mathrm{mg} / \mathrm{m}^{2}$ were considered to be at high risk of developing cardiotoxicosis ${ }^{17,18}$ and were automatically switched to dactinomycin to minimize any potential contribution of doxorubicin toxicity in assessing the toxicity of temozolomide and dacarbazine.

The maximum nonlethal dose of temozolomide in healthy male Beagles has been reported to be $200 \mathrm{mg} / \mathrm{m}^{2} .13$ We chose to use a dose of $60 \mathrm{mg} / \mathrm{m}^{2}$ as a starting point in the present study on the basis of available toxicology data for dogs, the minimum risk of toxicoses when administered at this dose in humans, and the convenience of administration given the currently available capsule sizes. In addition, we chose a conservative initial dose because this was, to our knowledge, the first evaluation of temozolomide in tumor-bearing dogs. Dose escalation in cohorts of 3 dogs resulted in a final median dose of $92.6 \mathrm{mg} / \mathrm{m}^{2}$.

One dog in the present study had episodes of urinary incontinence a few days after each cycle of temozolomide-dactinomycin administration was begun. These episodes lasted throughout the 5-day period of temozolomide administration during each treatment cycle. During each episode, results of a urinalysis and microbial culture of urine samples were negative. The dog was treated with antimicrobials each time, and signs regressed within 1 to 2 days after the last temozolomide dose was given in each cycle. In a study ${ }^{19}$ of 158 human patients treated for anaplastic astrocytoma with temozolomide as the sole agent, $13 \%$ of the patients experienced urinary incontinence. Thus, it is possible that neurologic toxicosis was the reason for the urinary incontinence in this dog.

Two dogs in the temozolomide-anthracycline treatment group in the present study developed azotemia during treatment. One dog had preexisting heart disease characterized by arrhythmias and was being treated with mexiletene, taurine, and enalapril. The dog had a complete response but became azotemic after the second treatment cycle. The dog was reevaluated echocardiographically and the dosage of enalapril was reduced. However, the azotemia progressed, and the owners elected to euthanize the dog. The other dog developed mild azotemia after the fifth treatment cycle. The azotemia did not progress in this dog, and the dog received an additional 3 cycles of treatment.

Results of the present study suggested that a combination of temozolomide and an anthracycline may be useful in the treatment of dogs with relapsed or refractory lymphoma. The greater ease of administration and lower toxicity were attractive features when temozolomide was compared with dacarbazine. Although duration of response was modest in the present study, 43 of $63(68 \%)$ dogs accrued had refractory disease that relapsed during the course of frontline doxorubicinbased induction therapy. These dogs would typically be expected to have shorter durations of response to rescue chemotherapy than the 3- to 5-month rescue remission durations expected for dogs that relapsed after completion of the induction chemotherapy protocol. ${ }^{5,20-24}$ In addition, because of the dose escalation aspect of the study, some dogs received relatively low doses of temozolomide. Although we did not find a significant relationship between temozolomide dose and duration of response to rescue chemotherapy, the statistical power for detecting such a relationship was low. Also, we used strict criteria for terminating the dose escalation of temozolomide because chemotherapy in these dogs was largely intended to be palliative. Additional dose escalation studies are needed to establish the maximum tolerated dose of temozolomide when administered in combination with anthracyclines in dogs with cancer. Treatment of dogs with lymphoma before refractory disease is established might also be expected to improve outcomes, and the addition of temozolomide to induction chemotherapy protocols merits further investigation.

a. Adriamycin, Bedford Laboratories Inc, Bedford, Ohio.

b. Cosmegen, Ovation Pharmaceuticals, Deerfield, Ill.

c. Dacarbazine, American Pharmaceutical Partners Inc, Schaumburg, Ill.

d. Temodar, Shering Corp, Kenilworth, NJ.

e. MedCalc for Windows, version 8.1.0.0, MedCalc Software, Mariakerke, Belgium.

\section{References}

1. Van Vechten M, Helfand SC, Jeglum KA. Treatment of relapsed canine lymphoma with doxorubicin and dacarbazine. J Vet Intern Med 1990;4:187-191.

2. Remmelink M, Salmon I, Delville JP, et al. In vitro characterisation of soft tissue tumor chemosensitivity. Anticancer Res 1997; 17:2009-2017.

3. Leikin S, Bernstein I, Evans A, et al. Use of combination adriamycin (NSC-123127) and DTIC (NSC-45388) in children with advanced stage IV neuroblastoma. Cancer Chemother Rep 1975;59:1015-1018.

4. Mellanby RJ, Herrtage ME, Dobson JM. Owners' assessments of their dog's quality of life during palliative chemotherapy for lymphoma. J Small Anim Pract 2003;44:100-103.

5. Ahaus EA, Couto CG, Valerius KD. Hematological toxicity of 
doxorubicin-containing protocols in dogs with spontaneously occurring malignant tumors. J Am Anim Hosp Assoc 2000;36: 422-426.

6. Gray KN, Raulston GL, Gleiser CA, et al. Histologic classification as an indication of therapeutic response in malignant lymphoma of dogs. J Am Vet Med Assoc 1984;184:814-817.

7. Stevens MF, Hickman JA, Stone R, et al. Antitumor imidazotetrazines. 1. Synthesis and chemistry of 8-carbamoyl-3-(2chloroethyl)imidazo[5,1-d]-1,2,3,5-tetrazin-4(3 H)-one, a novel broad-spectrum antitumor agent. J Med Chem 1984;27:196-201.

8. Tsang LL, Quarterman CP, Gescher A, et al. Comparison of the cytotoxicity in vitro of temozolomide and dacarbazine, prodrugs of 3-methyl-(triazen-1-yl)imidazole-4-carboxamide. Cancer Chemother Pharmacol 1991;27:342-346.

9. Stevens MF, Hickman JA, Langdon SP, et al. Antitumor activity and pharmacokinetics in mice of 8-carbamoyl-3-methyl-imidazo[5,1-d]-1,2,3,5-tetrazin-4(3H)-one (CCRG 81045; M \& B 39831), a novel drug with potential as an alternative to dacarbazine. Cancer Res 1987;47:5846-5852.

10. Reid JM, Kuffel MJ, Miller JK, et al. Metabolic activation of dacarbazine by human cytochromes P450: the role of CYP1Al, CYP1A2, and CYP2E1. Clin Cancer Res 1999;5:2192-2197.

11. Veterinary Cooperative Oncology Group. Common terminology criteria for adverse events (VCOGCTCAE) following chemotherapy or biological antineoplastic therapy in dogs and cats v1.0. Vet Comp Oncol 2004;2:195-213.

12. Kim HK, Lin CC, Parker D, et al. High-performance liquid chromatographic determination and stability of 5-(3-methyltriazen1-yl)-imidazo-4-carboximide, the biologically active product of the antitumor agent temozolomide, in human plasma. J Chromatogr B Biomed Sci Appl 1997;703:225-233.

13. Initial scientific discussion for the approval of Temodal. European Medicines Agency Web site. Available at: www.emea.europa. eu/humandocs/PDFs/EPAR/Temodal/274198en6.pdf. Accessed Jan 1, 2007.
14. Neff T, Beard BC, Peterson LJ, et al. Polyclonal chemoprotection against temozolomide in a large-animal model of drug resistance gene therapy. Blood 2005;105:997-1002.

15. Newlands ES, Blackledge GR, Slack JA, et al. Phase I trial of temozolomide (CCRG 81045: M\&B 39831: NSC 362856). Br J Cancer 1992;65:287-291.

16. Dhodapkar M, Rubin J, Reid JM, et al. Phase I trial of temozolomide (NSC 362856) in patients with advanced cancer. Clin Cancer Res 1997;3:1093-1100.

17. Loar AS, Susaneck SJ. Doxorubicin-induced cardiotoxicity in five dogs. Semin Vet Med Surg (Small Anim) 1986;1:68-71.

18. Hammer AS, Couto CG, Filppi J, et al. Efficacy and toxicity of VAC chemotherapy (vincristine, doxorubicin, and cyclophosphamide) in dogs with hemangiosarcoma. J Vet Intern Med 1991;5:160-166.

19. Yung WK, Prados MD, Yaya-Tur R, et al. Multicenter phase II trial of temozolomide in patients with anaplastic astrocytoma or anaplastic oligoastrocytoma at first relapse. Temodal Brain Tumor Group. J Clin Oncol 1999;17:2762-2771.

20. Calvert CA, Leifer CE. Doxorubicin for treatment of canine lymphosarcoma after development of resistance to combination chemotherapy. J Am Vet Med Assoc 1981;179:1011-1012.

21. Hohenhaus AE, Matus RE. Etoposide (VP-16). Retrospective analysis of treatment in 13 dogs with lymphoma. J Vet Intern Med 1990;4:239-241.

22. Moore AS, Ogilvie GK, Vail DM. Actinomycin D for reinduction of remission in dogs with resistant lymphoma. J Vet Intern Med 1994;8:343-344.

23. Rassnick KM, Mauldin GE, Al-Sarraf R, et al. MOPP chemotherapy for treatment of resistant lymphoma in dogs: a retrospective study of 117 cases (1989-2000). J Vet Intern Med 2002;16:576-580.

24. Alvarez FJ, Kisseberth WC, Gallant SL, et al. Dexamethasone, melphalan, actinomycin D, cytosine arabinoside (DMAC) protocol for dogs with relapsed lymphoma. J Vet Intern Med 2006;20:1178-1183. 